\title{
Impact of vaccination during pregnancy and staphylococci concentration on the presence of Bacillus cereus in raw human milk
}

\author{
Veronique Demers-Mathieu $\mathbb{I D}^{1} \cdot$ Gabrielle Mathijssen $^{1} \cdot$ Shawn Fels $^{1} \cdot$ Donald H. Chace $^{1} \cdot$ Elena Medo ${ }^{1}$
}

Received: 11 September 2019 / Revised: 10 December 2019 / Accepted: 21 December 2019 / Published online: 9 January 2020

(c) The Author(s), under exclusive licence to Springer Nature America, Inc. 2020

\begin{abstract}
Objective This study aimed to determine whether vaccination during pregnancy, prematurity, and staphylococci concentration influenced the presence of B. cereus or staphylococcal enterotoxins (SEs) in raw human milk from healthy mothers.

Study design Human milk samples were collected from 152 healthy women. B. cereus, S. aureus and coagulase-negative staphylococci (CNS) were enumerated using selective agar culture media. The detection of B. cereus spores and SEs were determined using ELISA.

Results CNS and B. cereus concentrations in milk from non-vaccinated mothers were higher than that from mothers vaccinated during pregnancy, but $S$. aureus did not differ. Prematurity did not affect $B$. cereus or staphylococci in human milk. S. aureus and CNS concentrations in human milk with the presence of B. cereus were higher than that with the absence of $B$. cereus. Viable B. cereus was present in $9.2 \%$ of raw human milk samples whereas SEs were not detected in any samples.

Conclusions Vaccination during pregnancy and low concentration of staphylococci could reduce the risk of B. cereus in raw human milk. The screening of $B$. cereus in raw human milk must be performed before pasteurization to reduce the risk of $B$. cereus infection in preterm infants.
\end{abstract}

\section{Introduction}

Preterm infants are at higher risk for infection than term infants due to their immature immune system and their conditions related to premature birth, including respiratory distress syndrome, chronic lung disease, retinopathy of prematurity [1]. In neonatal intensive care units (NICU), preterm infants are often fed donor human milk to supplement an insufficient maternal supply of mother's milk [2]. A potential source of infection in preterm infants may come from raw human milk or pasteurized donor milk products containing Bacillus cereus or staphylococcal enterotoxins [3-6]. The factors from maternal background that increases their presence in human milk remain unknown.

Veronique Demers-Mathieu

vdemers-m@medolac.com

1 Department of Neonatal Immunology and Microbiology, Medolac Laboratories A Public Benefit Corporation, Boulder City, NV, USA
B. cereus can induce infections that can lead to death in preterm infants [5-8]. B. cereus or Bacillus species was detected in $5.8 \%$ of 190 pasteurized donor milk samples [9] and 5\% of 303 pasteurized donor milk samples [10]. B. cereus concentration in Holder pasteurized donor milk was 3-fold higher than raw donor milk (unpasteurized), whereas shelf-stable donor milk (retort sterilization) had no colonies of B. cereus [11]. B. cereus spores in human milk resist pasteurization and their spores can change in vegetative cells and grow during the cooling and thawing steps to become active in pasteurized donor milk [12]. Commercial sterilization destroys $B$. cereus spores in human milk, which explains the absence of $B$. cereus vegetative cells and spores in retort donor milk product.

Testing for B. cereus before pooling and processing raw human milk is highly recommended, especially for pasteurized donor milk products [9]. The efficacy and optimal method to detect $B$. cereus in raw human milk is not well documented. Plate count agar, a non-selective culture media to enumerate total aerobic counts, was used after pasteurization of human milk to detect the presence of Bacillus species [10]. No selective agar culture media has been used 
to enumerate Bacillus cereus in raw or pasteurized human milk. Mannitol-egg yolk-phenol red-agar (MYP), a selective agar culture media, has been developed to enumerate vegetative cells and spores of $B$. cereus in foods containing other bacteria or microorganisms [13]. No study has used MYP agar to detect the presence of $B$. cereus in raw or pasteurized human milk or in fluid samples (bloods and stools) from infected infants. The lack of official method to detect $B$. cereus in raw or pasteurized human milk increases the risk of $B$. cereus infection in preterm infants.

$S$. aureus is another bacteria that was often related to infection in preterm infants hospitalized in NICU [14]. Although the pasteurization reduces $S$. aureus in human milk, some strains can produce heat-stable enterotoxins (SEs) [3], which are not eliminated by pasteurization. The $\mathrm{SE}$ concentration required in human milk to induce gastrointestinal illness (distended abdomen and bloody diarrhea) is extremely low ( $\approx 0.4-0.5 \mathrm{ng} / \mathrm{mL})$ [15] and may lead to mortality in vulnerable infants. The presence of SEs in raw human milk or pasteurized donor milk is not usually tested [9]. The factors from maternal background that affect the production of SEs by $S$. aureus are unknown.

This study aimed to determine whether vaccination during pregnancy, prematurity and staphylococci concentration influenced the presence of B. cereus or SEs in raw human milk from healthy mothers.

\section{Materials and methods}

Raw human milk samples were obtained from 152 healthy women through the Mother's Milk Cooperative (Boulder City, NV, USA). These women from the United States consented the use of their milk for research (no Institutional Review Board approval was needed). Mothers who were smokers, drug users or diagnosed with a systemic infection or other diseases (including obesity and human immunodeficiency virus) were excluded. The criteria pre-established for inclusion were passing blood test (negative tests for HIV, HTLV, hepatitis B or C and syphilis), living in the USA (residency), exclusively breastfeeding, completing health questionnaire questions and no use of specific medications. Milk samples (150-250 mL) were collected at home with clean electric breast pumps into sterile plastic containers and stored immediately at $-20{ }^{\circ} \mathrm{C}$ in deep freezers. The breast was cleaned with water on a washcloth (no soap or alcohol) before pumping. Milk samples were frozen and transported to Medolac Laboratories A Public Benefit Corporation where they were kept frozen until they were rapidly thawed to $37^{\circ} \mathrm{C}$. Batches (2-3 bags) from the same mother were pulled together to withdraw an aliquot $(100 \mathrm{~mL})$ of milk into a sterile tube for microbiological testing. After microbial sampling, two aliquots $(1 \mathrm{~mL})$ of each mother were added to sterile vials and were frozen at $-20{ }^{\circ} \mathrm{C}$ in an industrial freezer until the ELISA analysis (within one week).

For the enumeration of viable Bacillus cereus and staphylococci, raw human milk samples $(1 \mathrm{~mL})$ was added into $99 \mathrm{~mL}$ of peptone water $(0.1 \% \mathrm{w} / \mathrm{v})$. Diluted milk $(1 \mathrm{~mL})$ was added, in duplicate, to sterile plates to determine the counts of Bacillus cereus, Staphylococcus aureus and coagulasenegative staphylococci (CNS) using selective agar culture media. Baird-Parker (BP) agar base supplemented with 5\% egg yolk tellurite were used to enumerate $S$. aureus and CNS [16]. Mannitol-egg yolk polymyxin (MYP) agar supplemented with 25,000 units of polymyxin B (antimicrobic vial polymyxin B) and 5\% (v/v) of egg yolk enrichment $50 \%$ were used to enumerate $B$. cereus [13]. Selective agar culture media and supplements were purchased from Becton, Dickinson and Company (Spark, MD, USA). The protocols described by the manufacturer for the enumeration of $S$. aureus, CSN and B. cereus were followed appropriately. Staphylococcus species were incubated at $35^{\circ} \mathrm{C}$ for $48 \mathrm{~h}$ and $B$. cereus at $32^{\circ} \mathrm{C}$ for $48 \mathrm{~h}$ in aerobic conditions.

For the detection of Bacillus cereus spores, ELISAs were prepared as described in our previous studies [17, 18] with some modifications. ELISAs were recorded with a microplate reader SpectraMax iD5 (Molecular Devices, Sunnyvale, CA, USA) with two replicates of blanks, standards and samples. Raw human milk samples were centrifuged at $10,621 \times \mathrm{g}$ for $20 \mathrm{~min}$ at $4{ }^{\circ} \mathrm{C}$. After removing the fat layer with cotton swabs, the pellet and supernatant were mixed together and then separated into aliquots and stored at $-20{ }^{\circ} \mathrm{C}$ until analyzed (less than 2 weeks). Microplates (Clear Nunc MaxiSorp flat-bottom, Thermo Fisher Scientific, Rochester, NY, USA) were coated with $100 \mu \mathrm{L}$ of Bacillus spore Antigen Polyclonal Rabbit Antibody IgG (Thermo Fisher Scientific, Rockford, IL, USA) at $1.6 \mu \mathrm{g} /$ $\mathrm{mL}$ in $1 \times$ phosphate-buffered saline pH7.4 (PBS, Gibco, Grand Island, NY, USA). Microplates were incubated overnight at $4{ }^{\circ} \mathrm{C}$. After incubation, microplates were washed 3 times with PBS with $0.05 \%$ Tween-20 detergent (PBST) (Thermo Fisher Scientific) and then $200 \mu \mathrm{L}$ of blocking buffer (PBST with $0.05 \%$ of bovine serum albumin (BSA) fraction V (Roche Diagnostic GmbH, Manheim, Germany) was added in all wells for $1 \mathrm{~h}$ at room temperature. For positive controls, milk samples enumerated with MYP agar for B. cereus colonies were correlated to the B. cereus spore ELISA. The samples (pellet and supernatant) were diluted $2 \mathrm{x}$ with blocking buffer. For each step (addition of standards/controls/samples and secondary antibodies at $1 \mu \mathrm{g} / \mathrm{mL}$ ), washing and incubation for $1 \mathrm{~h}$ at room temperature were performed. Bacillus spore antigen polyclonal antibody conjugated with horseradish peroxidase (HRP) in PBS were used for detection. The substrate $(1 \times, 100 \mu \mathrm{L}), 3,3$ ',5,5'-tetramethylbenzidine 
(Invitrogen, Vienna, Austria) was added for $5 \mathrm{~min}$ at room temperature followed by addition of $50 \mu \mathrm{L}$ of $2 \mathrm{~N}$ hydrochloric acid to stop the coloration reaction. Optical density was measured at $450 \mathrm{~nm}$.

Concentration of staphylococcal enterotoxin A (SEA), B (SEB) and D (SED) were determined using ELISAs described previously [17, 18] with some modifications. Raw human milk samples were centrifuged at $1,301 \times \mathrm{g}$ for $20 \mathrm{~min}$ at $4{ }^{\circ} \mathrm{C}$. The infranate was collected, separated into aliquots and stored at $-20{ }^{\circ} \mathrm{C}$ until analyzed (less than 2 weeks). Plates were coated with $100 \mu \mathrm{L}$ of $1 \mu \mathrm{g} / \mathrm{mL}$ in $1 \times$ PBS of capture antibodies: sheep anti-SEA IgG for SEA, sheep antiSEB IgG for SEB and sheep anti-SED IgG for SED (Toxin Technology, Sarasota, FL, USA). Plates were incubated overnight at $4{ }^{\circ} \mathrm{C}$. After washing plates, $200 \mu \mathrm{L}$ of blocking buffer (PBST with $3 \%$ of BSA fraction V) was added in all wells for $1 \mathrm{~h}$ at room temperature. Standard samples were prepared using purified SEA, SEB and SED (Toxin Technology). The standard curves were prepared using a range from 0.1 to $50 \mathrm{ng} / \mathrm{mL}$. Supernatant samples were diluted $2 \times$ with blocking buffer for SEA, SEB and SED measurements. Sheep anti-SEA conjugated HRP, sheep anti-SEB conjugated HRP and sheep anti-SED conjugated HRP in blocking buffer were used to detect SEA, SEB and SED, respectively. Substrate, acid-reagent and reading steps were performed as previously described.

Detection of SEs using ELISA were validated for accuracy, precision, limit of detection, limit of quantification, lower limit of quantification (LLOQ) and upper limit of quantification (ULOQ). For these parameters, SEA, SEB and SED concentrations were determined in supernatant human milk with $25 \mathrm{ng} / \mathrm{mL}$ of SEA, SEB or SED in $2 \times$ blocking buffer from 4 days of experiments with three replicates.

For statistical analyses, unpaired $t$ tests in GraphPad Prism software (version 8) were used to compare factors from maternal background (vaccination during pregnancy: vaccinated vs unvaccinated; and prematurity: preterm-delivering vs term-delivering) for the concentrations of B. cereus, S. aureus and CNS in human milk. Differences were designated significant at $p<0.05$. Sample size ( $n=152$ mothers) was selected based on previous literature sample sizes and proved adequately powered based on the results.

\section{Results}

The demographic and perinatal characteristics for the women donating human milk are presented in Table 1. The number of vaccinated mothers and the type of vaccination are described in Table 1.

CNS and B. cereus concentrations in raw milk from unvaccinated mothers were 1.4 and 1.2-fold higher than in
Table 1 Demographic and perinatal characteristics.

\begin{tabular}{lll}
\hline & $\begin{array}{l}\text { Women } \\
(n=152)^{\mathrm{d}}\end{array}$ & $\begin{array}{l}\text { Presence of } B . \\
\text { cereus }^{\mathrm{b}}(n=14)\end{array}$ \\
\hline $\begin{array}{l}\text { Term-delivering: preterm- } \\
\text { delivering, } n\end{array}$ & $\begin{array}{l}134 \text { term: } 18 \\
\text { preterm }\end{array}$ & 13 term: 1 preterm \\
$\begin{array}{l}\text { Postpartum time, months } \\
\text { a }\end{array}$ & $\begin{array}{l}5 \pm 4(1-14) \\
74 \text { males: } 78\end{array}$ & $4 \pm 1(2-5)$ \\
Infant gender, $n$ & females \\
Mother's age, years & a 6 females \\
$\begin{array}{l}\text { Vaccinated during } \\
\text { pregnancy, } n(\%)^{\mathrm{c}}\end{array}$ & $98(64.5)$ & $4(28.6)$ \\
$\begin{array}{l}\text { Flu vaccine, } n(\%) \\
\text { Tdap vaccine, } n(\%)\end{array}$ & $25(25.2)$ & $1(7.1)$ \\
$\begin{array}{l}\text { Flu and Tdap vaccines, } \\
n(\%)\end{array}$ & $54(55.1)$ & $2(19.4)$ \\
$\begin{array}{l}\text { Unvaccinated during } \\
\text { pregnancy, } n(\%)\end{array}$ & $54(35.5)$ & $10(71.4)$ \\
\hline
\end{tabular}

${ }^{a}$ Value are mean \pm SD (min-max)

${ }^{\mathrm{b}}$ Mothers with the presence of Bacillus cereus in raw human milk

${ }^{c}$ Women were vaccinated with tetanus-reduced-dose diphtheria and acellular pertussis (Tdap) vaccine

and/or inactivated influenza (flu) vaccine between the second and third trimester (27-36 weeks of gestation)

${ }^{\mathrm{d}}$ Women took no medication (including over the counter, prescription medications and illicit substances) before and during the expression period

milk from mothers vaccinated during pregnancy (flu/inactivated influenza virus vaccine, tetanus, diphtheria and acellular pertussis (Tdap) vaccine and both flu and Tdap vaccines), respectively (Fig. 1). S. aureus concentration in raw human milk did not differ between vaccinated and unvaccinated women.

B. cereus, S. aureus, and CNS concentrations in raw human milk did not differ between preterm-delivering mothers and term-delivering mothers.

The concentrations of $S$. aureus and CNS in human milk with the presence of viable B. cereus were 16- and 2-fold higher than in human milk with the absence of $B$. cereus (Fig. 2).

Among 152 raw human milk samples, 9.2\% $(n=14)$ of them contained viable $B$. cereus enumerated on MYP whereas $21 \%(n=32)$ of milk samples contained B. cereus spores were detected via ELISA (Table 2). Among these 14 milk samples with the presence of viable B. cereus (MYP agar), $64.3 \%(n=9)$ of them contained $B$. cereus spores (ELISA).

No SE was detected in human milk samples, including those samples with high concentration of $S$. aureus. The limit of detection was $0.8 \mathrm{ng} / \mathrm{mL}$ for SEA, SEB and SED.

Table 3 summarizes the validation parameters for SEA, SEB and SED concentrations in raw human milk determined via ELISAs. The \% CV values calculated from the 

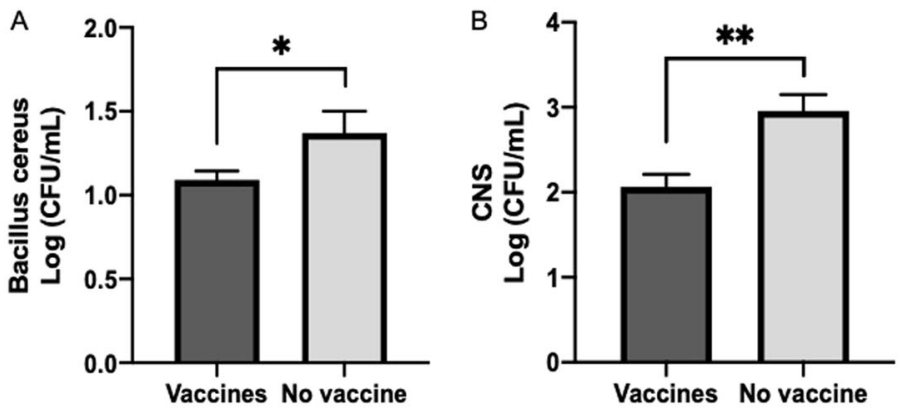

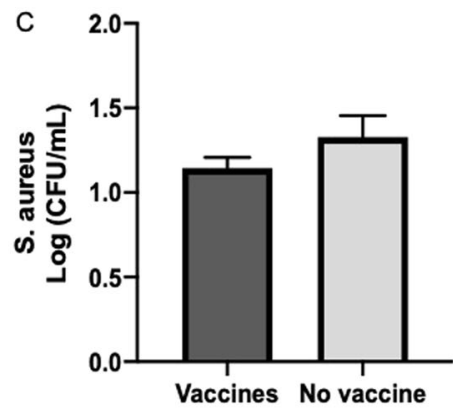

Fig. 1 Concentrations of viable a Bacillus cereus, b coagulasenegative staphylococci (CNS) and c Staphylococcus aureus in raw human milk between mothers vaccinated during pregnancy $(n=98)$ and unvaccinated mothers $(n=54)$. Values are mean \pm SEM. Asterisks

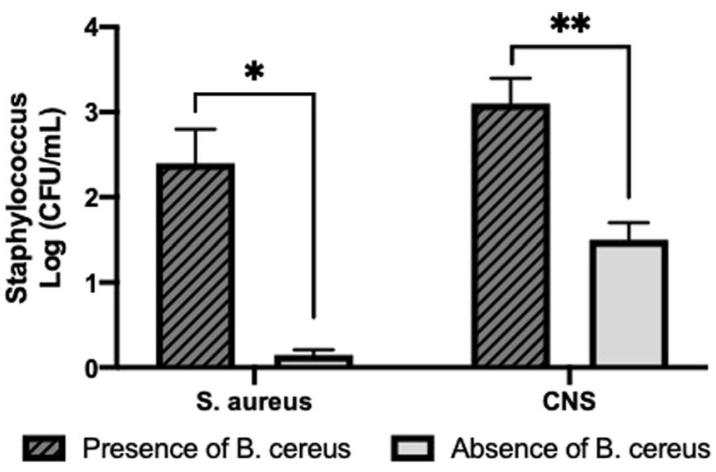

Fig. 2 Comparison of counts (colony forming units, Log CFU/mL) for Staphylococcus aureus and coagulase-negative staphylococcal between milk containing $B$. cereus (presence, $n=14$ ) and milk without $B$. cereus (absence, $n=125$ ). Values are mean \pm SEM. Asterisks show statistically significant differences between variables $(* * p<$ $0.01 ; *<0.05)$ using unpaired $t$-tests.

Table 2 Detection of Bacillus cereus spores in raw human milk using ELISA.

Optical density $^{\text {a }}$ Number of samples $(\mathrm{OD}, 450 \mathrm{~nm}) \quad(n=152)$

Pellet and supernatant milk

\begin{tabular}{lll} 
Presence of B. cereus spores & $0.62 \pm 0.05$ & $n=32$ \\
Absence of $B$. cereus spores & $0.03 \pm 0.01$ & $n=120$ \\
Controls for B. cereus spores & & \\
$<100 \mathrm{CFU} / \mathrm{mL}$ & $0.06 \pm 0.01$ & $n=8$ \\
$10,000 \mathrm{CFU} / \mathrm{mL}$ & $0.17 \pm 0.02$ & $n=8$ \\
$21,000 \mathrm{CFU} / \mathrm{mL}$ & $0.42 \pm 0.07$ & $n=8$ \\
\hline
\end{tabular}

${ }^{\mathrm{a}}$ Value are mean \pm SEM

${ }^{\mathrm{b}}$ Human milk controls were enumerated from MYP for viable $B$. cereus

analysis were $<9 \%$ for both intra-day (values for each SE in milk sample measured in triplicate) and inter-day assessments (average values of SE in milk across 4 days), which meets typical validation requirements $(<20 \%)$. show statistically significant differences between variables $(* * p<$ $\left.0.01 ;{ }^{*} p<0.05\right)$ using unpaired $t$-tests. Colony-forming units: CFU/ $\mathrm{mL}$. Pregnancy vaccination was the flu vaccine and/or tetanus, diphtheria and acellular pertussis (Tdap) vaccine.

Table 3 Validation for the detection of staphylococcal enterotoxins A (SEA), B (SEB) and D (SED) using ELISA ${ }^{c}$.

\begin{tabular}{llll}
\hline Parameters & SEA & SEB & SED \\
\hline Accuracy, \% & 7.8 & 12 & 10 \\
Precision, \%CV & 5.4 & 2.7 & 7.6 \\
limit of detection, ng/mL & 0.25 & 0.43 & 0.43 \\
limit of quantification, ng/mL & 0.54 & 2.4 & 0.41 \\
LLOQ $^{\text {a }}, \mathrm{ng} / \mathrm{mL}$ & 0.16 & 0.83 & 0.16 \\
$\mathrm{ULOQ}^{\mathrm{b}}, \mathrm{ng} / \mathrm{mL}$ & 200 & 200 & 200 \\
\hline
\end{tabular}

${ }^{\mathrm{a}} \mathrm{LLOQ}$, lower limit of quantification

${ }^{\mathrm{b}} \mathrm{ULOQ}$, upper limit of quantification

${ }^{\mathrm{c}}$ Validation values were from supernantant human milk sample containing $25 \mathrm{ng} / \mathrm{ml}$ of SEA, SEB or SED with 4 days and 3 replicated for each day

\section{Discussion}

The present study investigated the influence of factors from maternal background (pregnancy vaccination, prematurity and staphylococci concentration) on the presence of $B$. cereus and $S$. aureus SEs. B. cereus caused severe infections in preterm infants, including bacteremia, pneumonia and meningoencephalitis $[6,19,20]$. In all types of $B$. cereus disease, the virulence was associated with enterotoxin (cereulide) production: diarrheal syndrome (abdominal pain and diarrhea) is caused when vegetative cells of $B$. cereus produce cereulide in the small intestine, whereas emetic syndrome (nausea and vomiting) is caused by ingested food containing emetic B. cereus and/or preformed emetic cereulide [21]. The mode of transmission for B. cereus infection in preterm infants was reported to be from various sources (including ventilator equipment, staff hands and catheters), but many report cases did not find the origin of B. cereus contamination [6]. B. cereus strains were also isolated from gastric fluid in two preterm infants fed pasteurized donor milk and both samples (fluid and milk) had high virulence potency B. cereus [5]. These 
investigators suggested that pasteurized donor milk could be a reservoir for pathogenic B. cereus due to its higher growth in pasteurized milk [5]. Pasteurized donor human was also suspected to be the source for three cases of $B$. cereus infection in preterm infants [22]. Lewin et al. [23] reported that the association between $B$. cereus contaminated pasteurized donor milk and preterm infection has never been documented. The number of reported cases of $B$. cereus infections in infants, many of which were fatal, are likely to be underestimated due to the lack of microbiological methods that can be used to identify B. cereus in hospitals. Most Milk Banks do not have sufficient bacteriologic methods for the identification of $B$. cereus in human milk. The origin of $B$. cereus in raw or pasteurized human milk is still unclear.

For the first time, we demonstrated that B. cereus and CNS concentrations in raw human milk from mothers vaccinated during pregnancy (flu and/or Tdap vaccines) was lower than that from unvaccinated mothers. No previous study has examined the effect of vaccination during pregnancy on the microbial concentration in human milk. A possible explanation of the lower concentration of $B$. cereus and CNS in human milk from vaccinated mothers during pregnancy could be due to the higher level of human milk immune components (antibodies and immune cells) with antibacterial activity compared with that from nonvaccinated mothers. S. aureus did not differ between vaccinated mothers and non-vaccinated mothers, which could be related to lower milk immune components specific to $S$. aureus. Vaccination during pregnancy increases the secretion of pertussis-specific [24] and influenza-specific [25] antibodies in human milk, which may also provide passive protection against these pathogens. Therefore, flu and pertussis vaccines could increase the secretion of antibodies in human milk that are non-specific and prevent the growth of CNS and B. cereus, but no evidence has yet been provided. B. cereus, S. aureus and CNS concentrations in milk did not differ between preterm-delivering mothers and termdelivering mothers. This result is in accordance with Landers et al. [10] that found bacteria profiles of milk expressed from preterm-delivering mothers in the first month postpartum did not differ from that expressed by mothers delivering at full term. Vaccination during pregnancy is mandatory in some countries (France), whereas it is recommended (but not obligatory) by the FDA in USA. More studies are needed to evaluate the efficiency of vaccination on the prevention of pathogens in human milk.

We found that viable B. cereus was present in $9.2 \%$ of raw human milk samples expressed from 152 healthy women using MYP agar. This results is in agreement with two previous studies that found 5\% [10] and 5.8\% [9] of pasteurized donor milk samples with Bacillus species. However, these investigators did not perform a specific enumeration for Bacillus species or B. cereus and relied on the total aerobic counts using PCA [10]. Total aerobic counts in pasteurized human milk are mostly from Bacillus species because their spores survive pasteurization whereas other microorganisms are destroyed [26]. MYP agar is a selective and differential medium for the enumeration of spores and vegetative cells of $B$. cereus in the presence of high concentration of other bacteria in food due to its resistance to polymyxin, lack of mannitol fermentation (which produces pink red colonies) and presence of lecithinase (induce a zone of precipitate around the colonies) [13]. The presence of $B$. cereus spores was confirmed by ELISA on $64.5 \%$ of milk samples that were positive for $B$. cereus on MYP agar. ELISA was previously used for its high sensitivity detection of $B$. cereus spores in meat [27], but no previous study has used ELISA to detect $B$. cereus spores in human milk. Antibodies specific to $B$. cereus spore antigen can detect both dead and viable cells and spores, which may explain the higher number of milk samples that were positive for B. cereus spores $(26.7 \%)$. Therefore, MYP agar was more appropriate to detect $B$. cereus in raw human milk samples than ELISA due to its capacity to enumerate only viable active cells and spores from B. cereus.

B. cereus spores and vegetative cells in raw human milk are destroyed by commercial sterilization whereas the spores survive throughout pasteurization [12] and grow back (vegetative cells) in higher levels than in raw breast milk [11]. The faster growth of $B$. cereus in pasteurized donor milk samples could be partially due to the destruction of other microorganisms, which reduces the competition for nutrients. Lactoferrin and secretory IgA concentrations are reduced (66 and $26 \%$, respectively) after pasteurization of raw donor milk [28]. The lower concentration of these antimicrobial immune components in pasteurized donor milk is another factor that allows for faster growth of pathogenic bacteria [29]. Growth inhibition of $S$. aureus, E. coli or Pseudomonas aeruginosa was significantly lower in the pasteurized human milk compared with that in raw human milk [30]. Therefore, identification of Bacillus cereus before and after pasteurization is required to reduce the risk of $B$. cereus infection in preterm infants fed pasteurized human milk.

For the first time, we demonstrated that $S$. aureus and CNS concentrations in raw human milk were higher in the presence of viable $B$. cereus than in its absence. This result could be due to the interactions between $B$. cereus and staphylococci that stimulate $B$. cereus growth in human milk. Staphylococci could produce nutrients for B. cereus and/or inactive antimicrobial peptides/proteins by proteolysis. A previous study demonstrated that $B$. cereus stimulated enterotoxin production by $S$. aureus when grown at $25^{\circ} \mathrm{C}$ and $25^{\circ} \mathrm{C}$ in ham slurry [31], but no study has investigated the stimulation of $B$. cereus growth by 
staphylococci. The origin of Staphylococcus species in human milk is usually from the mother's skin and nipples [10], whereas B. cereus could come from water, dust or air [32]. S. aureus is the most often microorganism associated with the cause of mastitis in breastfeeding women [33]. Methicillin-resistant $S$. aureus in raw milk from mothers with mastitis was likely implicated in the mortality of preterm infants with sepsis [34]. Septicemia and necrotizing enterocolitis (NEC) in preterm infants was possibly related to raw human milk contaminated with CNS (identified $S$. epidermidis) [4]. S. epidermidis and S. aureus were also identified in blood from preterm infants fed pasteurized human milk [35]. Therefore, human milk containing high concentration of $S$. aureus or CNS ( $>10^{4} \mathrm{CFU} / \mathrm{mL}$ ) should be discarded (before pasteurization) to reduce the risk of infection in fragile and ill infants.

The presence of $B$. cereus in a few human milk samples was not likely related to uncleaned breast pumps (biofilms) and/or poor hygienic practices (washing hands and breasts) during milk collection. Each donor in this study was trained about how to clean their hands, breasts and breast-pump before collecting milk. For $71.4 \%$ of the raw human milk containing $B$. cereus, the $\mathrm{CFU} / \mathrm{mL}$ for Enterobacteriaceae (EB), total aerobic counts and $S$. aureus viable counts (data not shown) were lower than the limits of microbial contamination $\left(10^{4}, 10^{5}\right.$ and $10^{4}$, respectively) in raw human milk recommended by National Institute for Health and Care Excellence (NICE) clinical guideline [36] and by Lewis et al. [37].

The most common staphylococcal SEs associated with food poisoning are SEA, SEB and SED [38]. In the present study, SEA, SEB and SED were not detected in raw human milk, including those with high concentrations of $S$. aureus ( $>10^{5} \mathrm{CFU} / \mathrm{mL}$ ). This observation is in accordance with Almutawif et al. [39] that showed no production of SEA and SEB by $S$. aureus 2482 in raw human milk incubated at $4{ }^{\circ} \mathrm{C}$ for 10 days and $21^{\circ} \mathrm{C}$ for $18 \mathrm{~h}$. However, these investigators demonstrated that $S$. aureus produced SEA and SEB (limit of detection was $0.5 \mathrm{ng} / \mathrm{mL}$ ) in pasteurized donor milk incubated at $37^{\circ} \mathrm{C}$ for $9 \mathrm{~h}$ but no SEs were produced in raw human milk, suggesting that raw human milk can suppress production of SEs by $S$. aureus compared to pasteurized donor milk [39]. SEs were synthesized by $S$. aureus throughout the late logarithmic phase of growth or during the transition from the exponential to the stationary phase $\left(10^{8}-10^{9} \mathrm{CFU} / \mathrm{mL}\right)[39,40]$. SEA $(1 \mathrm{ng} / \mathrm{mL})$ was also detected in pasteurized cow's milk with $S$. aureus at $10^{4}$ $\mathrm{CFU} / \mathrm{mL}$ incubated at $35^{\circ} \mathrm{C}$ for $15 \mathrm{~h}$ [41]. In this study, $S$. aureus concentrations were below $10^{7} \mathrm{CFU} / \mathrm{mL}$ in all human milk samples, which could explain the absence of SEs. To verify this hypothesis, five raw human milk samples with high concentration of $S$. aureus were incubated at $35^{\circ} \mathrm{C}$ for $48 \mathrm{~h}$ and $72 \mathrm{~h}$ to reach exponential growth and no
SEs were detected (data not shown). Therefore, the absence of SEs could be due to the absence of $S$. aureus strain able to produce SEs or the inhibition of SE production by the milk microbiome or immune components with antimicrobial properties. Therefore, the risk of SEs in raw human milk is likely to be minimal, but their detection in pasteurized donor milk products is highly recommended to eliminate the small risk of heat-stable SEs remaining active after pasteurization.

A limitation of this study was that the virulence of $B$. cereus in raw breast milk was not investigated. Hemolysin $\mathrm{BL}$, nonhemolytic enterotoxin and cytotoxin $\mathrm{K}$ are the virulence factors usually involved in B. cereus foodborne diarrheal illness [42], but no study has identified a major factor for gastroenteritis caused by $B$. cereus. Future studies are needed to identify the prevalence of $B$. cereus cereulide in pasteurized human milk and the factors that induce B. cereus gastroenteritis in preterm infants. Another limitation was that we did not differentiate methicillin-resistant and methicillin-sensitive for $S$. aureus. Moreover, we did not have the exact date of vaccination during pregnancy for Tdap vaccine and/or flu vaccine, but only the approximately time (between second and third trimester).

Although pasteurization reduces the immune components (lactoferrin, antibodies and lysozyme) in raw human milk [43], pasteurized donor milk contains a critical source of active immune components, including lactoferrin, SIgA and oligosaccharides that are likely the main components for the prevention of NEC. Preterm infants fed raw human milk or pasteurized donor milk were associated with lower risk of NEC compared with those fed preterm formula, when given until 34 weeks of postpartum age [44]. Pasteurization of raw human milk also reduces the risk of postnatal cytomegalovirus (CMV) infection by its inactivation. Preterm infants fed raw human milk were associated with higher rate of postnatally acquired CMV infections compared with those fed pasteurized human milk [45]. However, these investigators also reported that preterm infants fed unpasteurized human milk had lower rate of NEC compared with those fed pasteurized human milk. Ultraviolet-C irradiation could be used to deactivate CMV in human milk [46]. Ultraviolet-C irradiation of human milk can preserve higher active immune proteins than Holder pasteurization, resulting in bacteriostatic properties similar to raw human milk [47]. Overall, raw and pasteurized human milk must be carefully analyzed for potential pathogens when used for feeding preterm infants.

\section{Conclusion}

The present study demonstrated that the vaccination during pregnancy could reduce the growth of B. cereus 
and CNS in raw human milk. The presence of B. cereus in raw human milk was associated with higher concentration of CNS and S. aureus compared with human milk without $B$. cereus. Microbial sampling on raw human milk samples before pooling and pasteurizing steps is highly recommended to discard those containing B. cereus or milk with too high level of staphylococci. The detection of $B$. cereus in human milk must be performed with specific and sensitive microbiological methods to reduce the risk of underestimating the presence of this pathogen that resists pasteurization. Improvement of the sensitivity of bacteriologic exams on human milk could be used to isolate the milk containing pathogenic and/or nonpathogenic species that can be harmful for immunosuppressed premature infants. Raw human milk is the gold standard for feeding term infants, but due to the immature immune system, preterm infants may need sterilized human milk supplemented with immune components to prevent microbial infection in NICU. Future studies are required to investigate cases of infections in preterm infants fed raw or pasteurized human milk to better understand the potential association with B. cereus. Further works are needed to determine whether the type and the time of vaccination during pregnancy influence the reduction of $B$. cereus and other pathogens in human milk.

Acknowledgements We thank all study participants for their valuable contributions. We also thank Adrianne Weir and Jennifer Medo for the recruitment of mothers and the management of milk samples.

Funding The authors disclosed receipt of the financial support from Medolac Laboratories A Public Benefit Corporation for the conduct of the study.

Author contributions Dr. VDM conceptualized and designed the study, carried out the analyses and the statistical analysis, drafted the manuscript, and approved the final paper as submitted. VDM have primary responsibility for the final content. GM carried out some ELISA analyses and EM conceptualized the study. GM, SF, DHC, and EM critically revised the paper and approved the final paper as submitted.

\section{Compliance with ethical standards}

Conflict of interest The authors are employees of Medolac Laboratories A Public Benefit Corporation.

Publisher's note Springer Nature remains neutral with regard to jurisdictional claims in published maps and institutional affiliations.

\section{References}

1. Schelonka RL, Infante AJ. Neonatal immunology. Semin Perinatol. 1998;22:2-14.
2. Carroll K, Herrmann KR. The cost of using donor human milk in the NICU to achieve exclusively human milk feeding through 32 weeks postmenstrual age. Breastfeed Med. 2013;8:286-90.

3. Novak FR, Almeida JA, Warnken MB, Ferreira-Carvalho BT, Hagler AN. Methicillin-resistant Staphylococcus aureus in human milk. Mem Inst Oswaldo Cruz. 2000;95:29-33.

4. Ng PC, Lewindon PJ, Siu YK, Wong W, Cheung KL, Liu K. Bacterial contaminated breast milk and necrotizing enterocolitis in preterm twins. J Hosp Infect. 1995;31:105-10.

5. Decousser JW, Ramarao N, Duport C, Dorval M, BourgeoisNicolaos N, Guinebretière $\mathrm{MH}$, et al. Bacillus cereus and severe intestinal infections in preterm neonates: Putative role of pooled breast milk. Am J Infect Control. 2013;41:918-21.

6. Hilliard NJ, Schelonka RL, Waites KB. Bacillus cereus bacteremia in a preterm neonate. J Clin Microbiol. 2003;41:3441-4.

7. Tokieda K, Morikawa Y, Maeyama K, Mori K, Ikeda K. Clinical manifestations of Bacillus cereus meningitis in newborn infants. $\mathrm{J}$ Paediatr Child Health. 1999;35:582-4.

8. John AB, Razak EA, Razak EE, Al-Naqeeb N, Dhar R. Intractable Bacillus cereus bacteremia in a preterm neonate. J Trop Pediatr. 2006;53:131-2.

9. Froh EB, Vanderpool J, Spatz DL. Best practices to limit contamination of donor milk in a milk bank. J Obstet Gynecol Neonatal Nurs. 2018;47:547-55.

10. Landers S, Undegrove K. Bacteriological screening of donor human milk before and after Holder pasteurization. Breastfeed Med. 2010;5:117-21.

11. Lima HK, Wagner-Gillespie M, Perrin MT, Fogleman AD. Bacteria and bioactivity in Holder pasteurized and shelf-stable human milk products. Curr Dev Nutr. 2017;1:e001438.

12. Cadot C, Tran SL, Vignaud ML, De Buyser ML, Kolst $\varnothing$ AB, Brisabois A, et al. InhA1, NprA, and HlyII as candidates for markers to differentiate pathogenic from nonpathogenic Bacillus cereus strains. J Clin Microbiol. 2010;48:1358-65.

13. Mossel DA, Koopman MJ, Jongerius E. Enumeration of Bacillus cereus in foods. Appl Environ Microbiol. 1967;15:650-3.

14. Layer F, Sanchini A, Strommenger B, Cuny C, Breier AC, Proquitté $\mathrm{H}$, et al. Molecular typing of toxic shock syndrome toxin-1and enterotoxin A-producing methicillin-sensitive Staphylococcus aureus isolates from an outbreak in a neonatal intensive care unit. Int J Med Microbiol. 2015;305:790-8.

15. Evenson ML, Hinds MW, Bernstein RS, Bergdoll MS. Estimation of human dose of staphylococcal enterotoxin A from a large outbreak of staphylococcal food poisoning involving chocolate milk. Int J Food Microbiol. 1988;7:311-6.

16. Chapin KC, Lauderdale TL Reagents, stains, and media: bacteriology. Manual of Clinical Microbiology, 8th ed. Am Soc Microbiol, Washington, DC. 2003: 354-93.

17. Demers-Mathieu V, Huston RK, Markell AM, McCulley EA, Martin RL, Spooner M, et al. Differences in maternal immunoglobulins within mother's own breast milk and donor breast milk and across digestion in preterm infants. Nutrients. 2019;11:920.

18. Demers-Mathieu V, Huston RK, Markell AM, McCulley EA, Martin RL, Dallas DC. Antenatal influenza A-Specific IgA, IgM, and IgG antibodies in mother's own breast milk and donor breast milk, and gastric contents and stools from preterm infants. Nutrients. 2019;11:1567.

19. Lequin MH, Vermeulen JR, Van Elburg RM, Barkhof F, Kornelisse RF, Swarte R, et al. Bacillus cereus meningoencephalitis in preterm infants: neuroimaging characteristics. Am J Neuroradiol. 2005;26:2137-43.

20. Jevon GP, Dunne jr WM, Hicks MJ, Langston C. Bacillus cereus pneumonia in premature neonates: a report of two cases. Pediatr infect Dis J. 1993;12:251-2. 
21. Ceuppens S, Rajkovic A, Heyndrickx M, Tsilia V, Van De Wiele $\mathrm{T}$, Boon $\mathrm{N}$, et al. Regulation of toxin production by Bacillus cereus and its food safety implications. Crit Rev Microbiol. 2011;37:188-213.

22. Rigourd V, Barnier JP, Ferroni A, Nicloux M, Hachem T, Magny $\mathrm{JF}$, et al. Recent actuality about Bacillus cereus and human milk bank: a new sensitive method for microbiological analysis of pasteurized milk. Eur J Clin Microbiol Infect Dis. 2018;37:1297-303.

23. Lewin A, Quach C, Rigourd V, Picaud JC, Perreault T, Frange P, et al. Bacillus cereus infection in neonates and the absence of evidence for the role of banked human milk: Case reports and literature review. Infect Control Hosp Epidemiol. 2019;40:787-93.

24. Halperin BA, Morris A, Mackinnon-Cameron D, Mutch J, Langley JM, McNeil SA, et al. Kinetics of the antibody response to tetanus-diphtheria-acellular pertussis vaccine in women of childbearing age and postpartum women. Clin Infect Dis. 2019;53:885-92.

25. Schlaudecker EP, Steinhoff MC, Omer SB, McNeal MM, Roy E, Arifeen SE, et al. IgA and neutralizing antibodies to influenza $\mathrm{A}$ virus in human milk: A randomized trial of antenatal influenza immunization. PloS ONE 2013;8:e70867.

26. Hanson ML, Wendorff WL, Houck KB. Effect of heat treatment of milk on activation of Bacillus spores. J Food Prot. 2005;68:1484-6.

27. Zhu L, He J, Cao X, Huang K, Luo Y, Xu W. Development of a double-antibody sandwich ELISA for rapid detection of Bacillus cereus in food. Sci Rep. 2016;6:16092.

28. Chang JC, Chen CH, Fang LJ, Tsai CR, Chang YC, Wang TM. Influence of prolonged storage process, pasteurization, and heat treatment on biologically-active human milk proteins. Pediatr Neonatol 2013;54:360-6.

29. Akinbi H, Meinzen-Derr J, Auer C, Ma Y, Pullum D, Kusano R, et al. Alterations in the host defense properties of human milk following prolonged storage or pasteurization. J Pediatr Gastroenterol Nutr. 2010;51:347-52.

30. Van Gysel M, Cossey V, Fieuws S, Schuermans A. Impact of pasteurization on the antibacterial properties of human milk. Eur $\mathbf{J}$ Pediatr. 2019;171:1231-7.

31. McCoy DW, Faber JE. Influence of food microorganisms on staphylococcal growth and enterotoxin production in meat. Appl Environ Microbiol. 1966;14:372-7.

32. Long SS, Prober CG, Fischer M Principles and practice of pediatric infectious diseases E-Book. Elsevier Health Sci; 2017.

33. Spencer JP. Management of mastitis in breastfeeding women. Am Fam Physician. 2008;78:727-31.

34. Gastelum DT, Dassey D, Mascola L, Yasuda LM. Transmission of community-associated methicillin-resistant Staphylococcus aureus from breast milk in the neonatal intensive care unit. Pediatr Infect Dis J. 2005;24:1122-4.
35. Cossey V, Vanhole C, Eerdekens A, Rayyan M, Fieuws S, Schuermans A. Pasteurization of mother's own milk for preterm infants does not reduce the incidence of late-onset sepsis. Neonatol. 2013;103:170-6.

36. Centre for Clinical Practice at NICE. Donor breast milk banks: The operation of donor milk bank services. UK, 2010. http://guida nce.nice.org.uk/cg93.

37. Lewin A, Delage, G, Bernier F, Germain M. Banked human milk and quantitative risk assessment of Bacillus cereus infection in premature infants: a simulation study. Can J Infect Dis Med Microbiol. 2019;2019:6348281.

38. Pinchuk IV, Beswick EJ, Reyes VE. Staphylococcal enterotoxins. Toxins 2010;2:2177-97.

39. Almutawif Y, Hartmann B, Lloyd M, Lai CT, Rea A, Geddes D. Staphylococcus aureus enterotoxin production in raw and pasteurized milk: The effect of selected different storage durations and temperatures. Breastfeed Med. 2019;14:256-61.

40. Czop JK, Bergdoll MS. Staphylococcal enterotoxin synthesis during the exponential, transitional, and stationary growth phases. Infect Immun. 1974;9:229-35.

41. Donnelly CB, Leslie JE, Black LA. Production of enterotoxin A in milk. Appl Environ Microbiol. 1968;16:917-24.

42. de Segura AG, Escuder D, Montilla A, Bustos G, Pallás C, Fernández $\mathrm{L}$, et al. Heating-induced bacteriological and biochemical modifications in human donor milk after holder pasteurisation. J Pediatr Gastroenterol Nutr. 2012;54:197-203.

43. Picaud JC, Buffin R, Picaud JC, Buffin R. Human milk-treatment and quality of banked human milk. Clin Perinat. 2017;44:95-119.

44. Sisk PM, Lambeth TM, Rojas MA, Lightbourne T, Barahona M, Anthony E, et al. Necrotizing enterocolitis and growth in preterm infants fed predominantly maternal milk, pasteurized donor milk, or preterm formula: a retrospective study. Am J Perinat. 2016;34:676-83.

45. Stock K, Griesmaier E, Brunner B, Neubauer V, KiechlKohlendorfer U, Trawöger R. Pasteurization of breastmilk decreases the rate of postnatally acquired cytomegalovirus infections, but shows a nonsignificant trend to an increased rate of necrotizing enterocolitis in very preterm infants-a preliminary study. Breastfeed Med. 2015;10:113-7.

46. Lloyd ML, Hod N, Jayaraman J, Marchant EA, Christen L, Chiang $\mathrm{P}$, et al. Inactivation of cytomegalovirus in breast milk using ultraviolet-C irradiation: opportunities for a new treatment option in breast milk banking. PLoS ONE 2016;11: e0161116.

47. Christen L, Lai CT, Hartmann B, Hartmann PE, Geddes DT. The effect of UV-C pasteurization on bacteriostatic properties and immunological proteins of donor human milk. PLoS ONE 2019;8: e85867. 